\section{Development of Controlled- Release Solid Dispersion Granules Containing a Poorly Water-Soluble Drug, Porous Calcium Silicate, and the Water-Soluble Polymer Polyvinylpyrrolidone}

\begin{abstract}
Objectives: This study sought to develop controlled-release solid dispersion granules containing a poorly water-soluble drug, porous calcium silicate (PCS), and a water-soluble polymer.

Methods: Nifedipine (NIF) and indomethacin (IND), and several water-soluble polymers were selected to prepare solid dispersion granules. Differential scanning calorimetry, Powder X-ray diffraction, and Fourier transform infrared (FT-IR) were used to estimate the crystallinity of drugs in the PCS granules and to determine interactions between drug and PCS.

Results: Among the water-soluble polymers used in this study, only polyvinylpyrrolidone (PVP) permitted sustained release from the NIF granules. The release rates of NIF from the granules decreased as the molecular weight or amount of PVP in the granules was increased. A significant loss of crystallinity of NIF in the granules and the existence of a hydrogen bond between NIF and PCS or PVP, as well as between PCS and PVP were observed. To examine the applicability of this system to other drugs, indomethacin (IND) was selected. However, IND was rapidly released from the PVP-containing granules. FT-IR spectroscopy results revealed the existence of a hydrogen bond between IND and PVP, but IND and PCS appeared to interact via salt formation.
\end{abstract}

Conclusion: Hydrogen bonding among the drugs, PCS, and PVP may have contributed to the slow drug release in the developed system.

Keywords: Poorly water-soluble drug; Controlled release; Porous Calcium silicate; PVP K-90; Solid dispersion; Nifedipine

\section{Introduction}

According to the biopharmaceutical classification system (BCS), class II compounds exhibit low solubility and high permeability due to their hydrophobic characteristics, which result in poor bioavailability after oral administration [1]. Approximately $46 \%$ of newly discovered or investigational drugs fall into the BCS class II category [2,3]. Thus, the development of an oral administration system to increase their solubility remains a challenging task for pharmaceutical scientists. Various techniques, such as particle size reduction, crystal habit modification, complexation, solubilization, solid dispersion in carriers, and salt formation, are employed to improve the aqueous solubility of class II drugs [4]. Solid dispersion has been achieved via hot-melt extrusion, supercritical fluid, and cryogenic freezing

\section{Journal of}

Pharmaceutics \& Pharmacology

\section{Yuko Uegaki, Nobuaki Hirai, Tomoka Takatani- Nakase and Koichi Takahashi*}

Department of Pharmaceutics, School of Pharmacy and Pharmaceutical Sciences, Mukogawa Women's University, Hyogo, Japan

\section{Address for Correspondence}

Koichi Takahashi, Department of Pharmaceutics, School of Pharmacy and Pharmaceutical Sciences, Mukogawa Women's University, Hyogo, Japan, Email: koichi@mukogawa-u.ac.jp

Submission: 14 August, 2018

Accepted: 17 September, 2018

Published: 27 September, 2018

Copyright: () 2018 Uegaki $\mathrm{Y}$, et al. This is an open access article distributed under the Creative Commons Attribution License, which permits unrestricted use, distribution, and reproduction in any medium, provided the original work is properly cited.

technologies [5-8]. However, these technologies have some limitations for scale-up [9-12]. Meanwhile, the solvent evaporation method and the spray-drying method are the manufacturing technique used to achieve solid dispersion $[13,14]$.

Soliddrugdispersionsaregenerallypreparedviadispersioninawatersoluble carrier. Hydroxypropyl methylcellulose (HPMC), poloxamers, polyvinylpyrrolidone (PVP), hydroxypropyl methylcellulose acetate succinate (HPMCAS) and poly(vinylpyrrolidone-co-vinyl acetate (PVPVA) are the most widely used solid dispersion carriers because of their strong hydrophilic properties and ability to form molecular adducts with many compounds [15-18]. Unlike conventional solid dispersion, the carriers used in surface solid dispersion are water insoluble, porous materials with hydrophilic properties. Silica gel and calcium silicate have extremely large surface areas, which are attributed to their highly porous structure, and they have been reported to improve the solubility of poorly water-soluble drugs [1923]. Porous calcium silicate (PCS) has a highly porous structure and a large individual pore volume, and it has been used as a liquid absorber and a compressive adjuvant of powder for tableting [24]. PCS is also used as a carrier for solid dispersion to improve the dissolution of poorly water-soluble drugs [21-23]. We reported the development of solid dispersion tablets via a simple wet granulation method using PCS $[25,26]$.

Conversely, for BCS Class II drugs with short half-lives, it may be desirable to prepare controlled release compositions [27]. Controlled release of drugs from solid dispersions may also be advantageous for delivering drugs to the small intestines, and reducing the rate of drug release in the lumen may lower the extent of supersaturation. Controlled-release amorphous solid dispersions must also be formulated to prevent drug precipitation within the dosage form to ensure the drug is liberated from the dosage form following administration.

To prepare controlled-release solid dispersion formulations for BCS Class II drugs, different polymers, including both hydrophobic (e.g. ethyl cellulose, amino methacrylate copolymer) and hydrophilic 
Citation: Uegaki Y, Hirai N, Takatani-Nakase T, Takahashi K. Development of Controlled-Release Solid Dispersion Granules Containing a Poorly WaterSoluble Drug, Porous Calcium Silicate, and the Water-Soluble Polymer Polyvinylpyrrolidone. J Pharmaceu Pharmacol. 2018; 6(1): 7.

(e.g. hydroxylpropyl cellulose (HPC), hydroxylpropyl methyl cellulose (HPMC), methylcellulose), have been used [28-31]. In the polymer matrix system, the drug is homogeneously distributed throughout a matrix, and drug release is controlled by water-swellable or hydrophobic polymeric excipients. Silica has also been used to prepare controlled-release solid dispersion formulations, and a hydrophobic polymer is usually used jointly to control drug release [32].

To our knowledge, only a few reports have investigated the preparation of controlled-release solid dispersion granules using only water-soluble polymers and silica. Thus, this study aimed to develop and evaluate novel controlled-release solid dispersion granules. Nifedipine (NIF) and PCS were used as the poorly water-soluble drug and carrier, respectively, and solvent evaporation and wet granulation methods were used to prepare the granules. It is well known that dissolution from solid dispersion granules containing NIF and PVP or PCS is extremely rapid [25,33]. Initially, PCS granules containing a water-soluble polymer and NIF were prepared and evaluated. Secondly, granules containing indomethacin (IND), PVP, and PCS were prepared and compared with those containing NIF, PVP, and PCS.

\section{Materials and Methods Materials}

NIF and IND (both purity $>99.0 \mathrm{w} / \mathrm{w} \%$ ) were purchased from Permachem Asia Ltd. (Tokyo, Japan) and Kongo Chemical Co. Ltd. (Toyama, Japan), respectively. PCS (Fluorite RE) was obtained from Tomita Pharmaceutical Co. Ltd. (Tokushima, Japan). HPMC (Metolose 60SH-10000), Polyvinylpyrrolidone-vinyl acetate copolymers (Kollidon VA64; PVP/VA), Hydroxypropyl cellulose (NISSO HPC H), and polyvinyl alcohol (Gohsenol" EG-40; PVA) were purchased from Shin-Etsu Chemical Co. Ltd. (Tokyo, Japan), BASF Japan Co. Ltd. (Tokyo, Japan), Nippon Soda Co. Ltd. (Tokyo, Japan), and Nippon Synthetic Chemical Industry Co. Ltd. (Osaka, Japan), respectively. Xylitol which was used as a binder, was obtained from B Food Science Co. Ltd. (Tokyo, Japan). PVP K25 and PVP K90 were purchased from Nacalai Tesque Ltd. (Kyoto, Japan). PVP K60 was obtained from Tokyo Chemical Industry Co. Ltd. (Tokyo, Japan). All other chemicals were of reagent grade and were used without further purification.

\section{Preparation of solid dispersion granules}

NIF (5 g) or IND (10 g) and an aqueous polymer (20 g) were dissolved in an appropriate amount of ethanol $(100 \mathrm{~g})$ via heating at $60{ }^{\circ} \mathrm{C}$. The solution was added to PCS $(40 \mathrm{~g})$ and mixed for 15 min using a high-speed agitation granulator (High-Speed Mixer, Earthtechnica Co. Ltd., Tokyo, Japan) at $250 \mathrm{rpm}$ with an agitator and $2500 \mathrm{rpm}$ with a chopper. After drying at $70{ }^{\circ} \mathrm{C}$ for $12 \mathrm{~h}$, Xylitol (70 g) in distilled water $(70 \mathrm{~g})$ was added to this dried mixture to prepare the granules using the granulator at $250 \mathrm{rpm}$ with an agitator and 2500 rpm with a chopper, and the granulation endpoint was determined visually. The granules were dried at $70{ }^{\circ} \mathrm{C}$ for $12 \mathrm{~h}$. Then, the granules were pulverized in a speed mill (Okada Seiko Co. Ltd., Tokyo, Japan). Granules ranging in size from 500 to $850 \mu \mathrm{m}$ were used in this study. To prepare granules using the dry method, the aforementioned dried mixture $(60 \mathrm{mg})$ and xylitol $(64.6 \mathrm{mg})$ were mixed, and this mixture was compressed at $20 \mathrm{MPa}$ using 14-mm flat face punches (SSP-10A, Shimadzu Corp., Kyoto, Japan) on a single punch. After pulverization, granules ranging in size from 500 to $850 \mu \mathrm{m}$ were obtained.

\section{Preparation of the adsorption solid dispersion and physical} mixture

NIF $(0.5 \mathrm{~g})$ or IND $(1 \mathrm{~g})$ and PVP $(2 \mathrm{~g})$ were dissolved in ethanol $(10 \mathrm{~g})$ via heating at $60^{\circ} \mathrm{C}$, and then this solution was added to PCS (4 g) and mixed for 15 min using a rotation mixer. The mixture was dried for $12 \mathrm{~h}$ at $70{ }^{\circ} \mathrm{C}$. The physical mixture (PM) was prepared by mixing NIF (0.5 g) or IND (1 g), PVP (2 g), and PCS (4 g) using a spatula.

\section{Drug release experiments}

Dissolution tests were performed according to the JP17 paddle method using Riken's Dissolution Tester (Miyamoto Riken Ind Co. Ltd., Osaka, Japan). Granules containing $10 \mathrm{mg}$ of NIF or IND were added to the dissolution medium $(900 \mathrm{ml}$ of purified water) at $37{ }^{\circ} \mathrm{C} \pm 0.5^{\circ} \mathrm{C}$, and the paddle was rotated at $50 \mathrm{rpm}$. The amount of dissolved NIF or IND was analyzed using an ultraviolet spectrophotometer (UV-1200, Shimadzu Corp.) at 350 or $320 \mathrm{~nm}$, respectively. The NIF and IND content of the granules were calculated using an ultraviolet spectrophotometer. An adequate amount of granules equivalent to $10 \mathrm{mg}$ of NIF or IND was accurately weighed, dissolved, and suitably diluted in methanol and measured using an ultraviolet spectrophotometer at 350 or $320 \mathrm{~nm}$.

\section{Kinetics of drug release}

Drug release kinetics was investigated by employing the empirical equation proposed by Korsmeyer-Peppas and Ritger-Peppas to analyze both Fickian and non-Fickian drug release from swelling as well as non-swelling polymeric delivery systems as follows [34-36] [Eqn 1]:

$$
\frac{M t}{M \infty}=k t^{n}
$$

Where $M t$ and $M \infty$ are the absolute cumulative amount of drug released at time and the maximum amount released in the experimental conditions employed at the plateau of the release curves, respectively. Therefore, $M t / M \infty$ represents the proportion of drug released at time $t . k$ is a kinetic constant related to the drug and structural and geometric properties of the micro particles, and $n$ is the release exponent, which is indicative of the release mechanism of the drug through the polymer matrix network. The log value of the drugdissolved fraction was plotted against log time to determine the drug release exponent (Eqn 2).

$$
\log \left(\frac{M t}{M \infty}\right)=\log k+n \log t
$$

\section{Fourier transform infrared spectroscopy}

IR spectra of powder samples were obtained using a spectrophotometer (IRAffinity-1, Shimadzu Corp.) using the potassium bromide $(\mathrm{KBr})$ pellet method. $\mathrm{KBr}$ disks were prepared by mixing several milligrams of the sample with $\mathrm{KBr}$ and compacting. The scan range was $400-4000 \mathrm{~cm}^{-1}$.

\section{Powder X-ray diffraction analysis}

PXRD analysis was performed using $\mathrm{Cu}$ K-ALPHA1 radiation, a voltage of $40 \mathrm{kV}$, and a current of $200 \mathrm{~mA}$ (RINT-2000, Rigaku Corporation, Tokyo, Japan). The sample was mounted in a flat sample 
Citation: Uegaki Y, Hirai N, Takatani-Nakase T, Takahashi K. Development of Controlled-Release Solid Dispersion Granules Containing a Poorly WaterSoluble Drug, Porous Calcium Silicate, and the Water-Soluble Polymer Polyvinylpyrrolidone. J Pharmaceu Pharmacol. 2018; 6(1): 7.

holder. The sampling interval was $0.02^{\circ}$, and the scan rate was $5^{\circ} / \mathrm{min}$ over a $2 \theta$ range of $5^{\circ}-70^{\circ}$.

\section{Differential scanning calorimetry of granules}

DSC was performed using a DSC-60 Plus (Shimadzu Corp.) to determine the physical state of NIF. Samples (1-10 mg) were sealed in an aluminum pan, and the heating curves were recorded using a constant heating rate of $5{ }^{\circ} \mathrm{C} / \mathrm{min}$ from $30^{\circ} \mathrm{C}$ to $200{ }^{\circ} \mathrm{C}$.

\section{Statistical analysis}

All results are presented as the mean \pm SD. Statistical differences were determined via the Turkey-Kramer nonparametric comparison test using Statcel for Windows. $\mathrm{P}<0.05$ denoted a statistically significant difference for all statistical tests used.

\section{Results and Discussion}

\section{Effect of aqueous polymers on the dissolution of NIF}

Chemical structures of model drugs (NIF and IND) are shown in Figure 1.

In this study, HPMC, HPC, PVA, PVP K-90, and PVP/VA were selected as the aqueous polymers, and their effects on the dissolution rate of NIF from solid dispersion granules were examined, as shown in Figure 2. Although prolonged NIF release was achieved with all polymers, the effects were greatest with PVP K-90. Thus, PVP was selected as an aqueous polymer.

\section{Effects of PVP on the dissolution of NIF from granules}

The effects of the molecular weight of PVP on the dissolution of NIF from granules were investigated. The suppressive effects of PVP on the release rate of NIF increased as its molecular weight was increased (Figure 3). From these results, PVP K-90 was selected as the excipient for sustained-release formulations. To investigate the effect of the amount of PVP K-90, various formulations were prepared, and

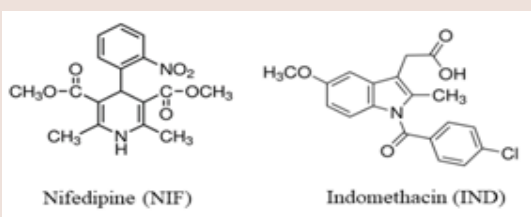

Figure 1: Chemical structures of model drugs.

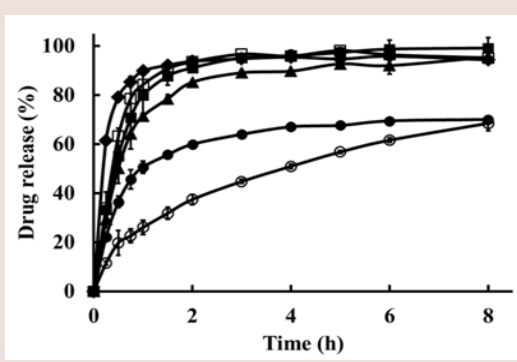

Figure 2: Effects of the polymer on the dissolution of nifedipine (NIF) from granules: hydroxypropyl methylcellulose (HPMC, $\bullet$ ), hydroxypropyl cellulose (HPC, A), polyvinyl alcohol (PVA, -), polyvinylpyrrolidone (PVP) K-90 (०), polyvinylpyrrolidone-vinyl acetate copolymers (PVP/VA, $\Delta$ ), and no polymer ( $\square)$. Data are presented as the mea $\pm \operatorname{SD}(n=3-4)$

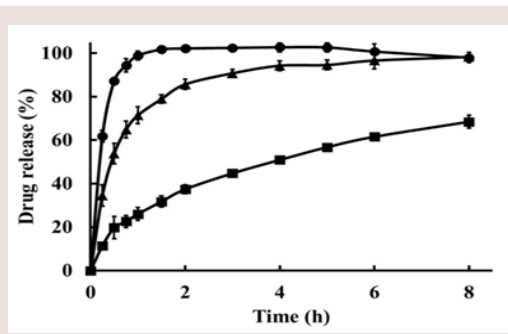

Figure 3: Effect of the molecular weight of polyvinylpyrrolidone (PVP) on the dissolution of nifedipine (NIF) from the granules. K-25 (•), K-60 ( $\Delta$ ), and K-90 (घ). Data are presented as the mean \pm SD $(n=3-6)$.

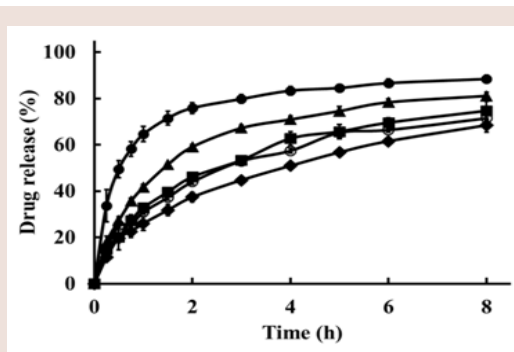

Figure 4: Effect of the amount of polyvinylpyrrolidone (PVP) on the dissolution of nifedipine (NIF) from the granules. PVP K-90/NIF (w/w): $0.5(\bullet), 1(\boldsymbol{\Delta}), 1.5$ $(\square), 2(0)$, or $4(\bullet)$. Data are presented as the mean \pm SD $(n=4-8)$.

Table 1: Compositions of nifedipine formulations

\begin{tabular}{|l|c|c|c|c|c|}
\hline \multirow{2}{*}{} & \multicolumn{5}{|c|}{ NIF/PVP (w/w) } \\
\cline { 2 - 6 } & $\mathbf{0 . 5}$ & $\mathbf{1}$ & $\mathbf{1 . 5}$ & $\mathbf{2}$ & $\mathbf{4}$ \\
\hline NIF (g) & 5 & 5 & 5 & 5 & 5 \\
\hline PVP (g) & 2.5 & 5 & 7.5 & 10 & 20 \\
\hline PCS (g) & 40 & 40 & 40 & 40 & 40 \\
\hline Xylitol (g) & 70 & 70 & 70 & 70 & 70 \\
\hline Ethanol (g) & 100 & 100 & 100 & 100 & 100 \\
\hline Water (g) & 75 & 75 & 75 & 75 & 75 \\
\hline
\end{tabular}

NIF: Nifedipine; PVP: Polyvinylpyrrolidone K-90; PCS: Porous Calcium Silicate

dissolution studies were conducted (Table 1). The dissolution rate declined as the amount of PVP K-90 was increased (Figure 4).

The NIF release data were analyzed using Eqn 2 to obtain the release exponent $\mathrm{n}$ and kinetic constant $\mathrm{k}$. A good linear relationship was observed between $\mathrm{n}$ and time, and the results are presented in Table 2. $\mathrm{n}<0.43$ indicates Fickian diffusion release from a spherical sample. Meanwhile, $0.43<\mathrm{n}<0.85$ indicates a non-Fickian mechanism, an anomalous behavior corresponding to polymer hydration, solvent penetration, drug dissolution, and polymer erosion. A value of $\mathrm{n}=0.85$ indicates a zero-order release mechanism (case II transport mechanism) [37-40]. The values of $n$ ranged 0.49-0.59 in this study. Thus, the release mechanism from the NIF granules may be nonFickian. Thus, it appears that PVP hydration, water penetration, and PVP erosion affected NIF release from the granules. The values of $\mathrm{k}$ decreased as the PVP/NIF ratio was increased (Figure 5). From these results, it may be possible to control the rate of drug release from the granules based on the PVP K-90 content.

\section{Evaluation of NIF in PCS formulations containing PVP-K90}

PXRD patterns were examined to investigate the effect of PVP 
Citation: Uegaki Y, Hirai N, Takatani-Nakase T, Takahashi K. Development of Controlled-Release Solid Dispersion Granules Containing a Poorly WaterSoluble Drug, Porous Calcium Silicate, and the Water-Soluble Polymer Polyvinylpyrrolidone. J Pharmaceu Pharmacol. 2018; 6(1): 7.

ISSN: $2327-204 X$

Table 2: Kinetic parameters of nifedipine release from the granules.

\begin{tabular}{|l|c|c|c|}
\hline PVP/NF (w/w) & $\mathbf{n}$ & $\mathbf{k}$ & $\mathbf{R}^{\mathbf{2}}$ \\
\hline 4 & $0.5152 \pm 0.055$ & $0.256 \pm 0.024$ & $0.9794 \pm 0.031$ \\
\hline 2 & $0.4885 \pm 0.047$ & $0.3059 \pm 0.015$ & $0.9337 \pm 0.031$ \\
\hline 1.5 & $0.5376 \pm 0.051$ & $0.3115 \pm 0.015$ & $0.9676 \pm 0.031$ \\
\hline 1 & $0.5854 \pm 0.072$ & $0.4078 \pm 0.015$ & $0.9944 \pm 0.031$ \\
\hline 0.5 & $0.5824 \pm 0.04$ & $0.6768 \pm 0.015$ & $0.9845 \pm 0.028$ \\
\hline
\end{tabular}

NIF: Nifedipine; PVP: Polyvinylpyrrolidone K-90; Data are Mean \pm SD $(n=3-8)$.

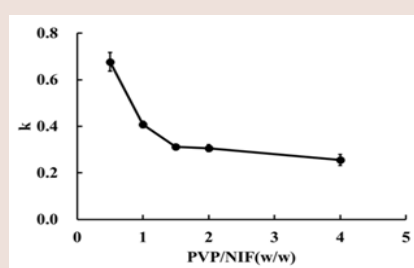

Figure 5: Effect of the amount of polyvinylpyrrolidone (PVP) on the kinetic constant in Eqn 1. Data are presented as the mean $\pm \mathrm{SD}(n=3-8)$. (a)

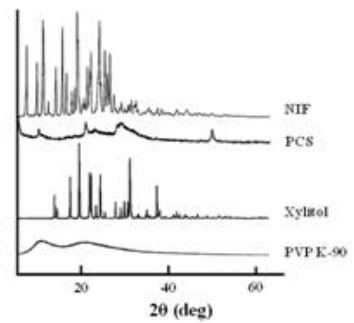

(b)

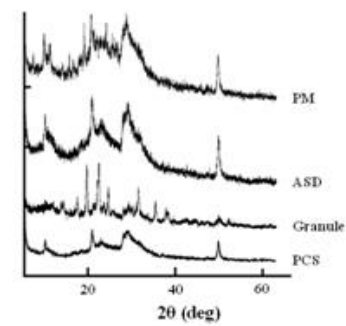

Figure 6: Powder X-ray diffraction (PXRD) patterns of (a) Nifedipine (NIF), porous calcium silicate (PCS), xylitol, and polyvinylpyrrolidone (PVP) K-90. (b) Physical mixture (PM) (NIF, PVP, PCS and xylitol; 1:4:8:14), adsorption solid dispersion (ASD), and granules.

K-90 in PCS formulations on the crystallinity of NIF. The presence of multiple peaks in the diffraction of NIF (Figure 6a) indicated a high degree of crystallinity of NIF, and the representative peaks were observed at $8^{\circ}$ and $16^{\circ}$. By contrast, PCS and PVP K-90 exhibited a low degree of crystallinity. The characteristic peaks of the PM were lower in intensity, indicating a dilution effect due to the addition of PCS and/ or PVP K-90. Meanwhile, the characteristic peaks of NIF disappeared in the ASD and granules (Figure 6b), suggesting that NIF has partial amorphous states in the ASD and granules. As the wet granulation method was used to prepare granules in this study, it appears that the wet granulation process affects the crystallinity of NIF.

Figure 7 shows the DSC thermograms of different NIF samples. NIF exhibited a melting endotherm at $175.2{ }^{\circ} \mathrm{C}$. Figures $7 \mathrm{a}$ and $7 \mathrm{~b}$ presents the DSC thermograms of the PM and ASD containing NIF and PVP K-90 (1:4) or PCS (1:8), respectively. Figure 7c presents the DSC of the PM and ASD containing NIF, PVP K-90, and PCS (1:4:8). Regarding the PM samples, the endothermic peaks of NIF were observed, and a slight decrease in the melting temperature of NIF was observed due to dilution of the drug. However, endothermic peaks were not observed for the ASD samples. Moreover, the endothermic peaks of the PM containing NIF, PVP K-90, PCS, and xylitol (1:4:8:14) were observed, but the peak of the granules containing NIF, PVP K-90, PCS, and xylitol (1:4:8:14) was not observed (Figure 7d).
FT-IR spectra were obtained to assess interactions among NIF, PVP K-90, and PCS (Figure 8). The spectrum of pure NIF (Figure 8a) displayed characteristic peaks at $1678(\mathrm{C}=\mathrm{O}$ stretching $)$ and 3327 $\mathrm{cm}^{-1}$ (secondary-NH). Figures $8 \mathrm{~b}$ and $8 \mathrm{c}$ presents the spectra of the PM and ASD containing NIF and PVP K-90 (1:4) or PCS (1:8). The characteristic peaks of NIF were observed for the PM although they were somewhat smaller. However, these peaks were observed rarely for the ASD. Therefore, it was revealed that $\mathrm{C}=\mathrm{O}$ and $\mathrm{NH}$ groups are involved in the interaction of NIF with PVP K-90 or PCS. Sharma S et al. and Kinoshita $\mathrm{M}$ et al. reported that the presence of hydrogen bonding between the silanol group of PCS and the $\mathrm{C}=\mathrm{O}$ groups of drugs $[22,23]$. Furthermore, hydrogen bonding between the N-H groups of NIF and PVP has been described [41,42]. From these results, the decreased crystallinity of NIF might be associated with hydrogen bonding between NIF and PVP K-90 or PCS. Furthermore, the same results were obtained for the PM and ASD of NIF, PVP K-90, and PCS (1:4:8, Figure $8 \mathrm{~d}$ ) and the PM and granules of NIF, PVP K-90, PCS, and xylitol (1:4:8:14, Figure 8e). These results suggested that NIF existed in the amorphous state in granules prepared via wet granulation.

\section{IND release in the PCS/PVP K-90 granules}

We previously reported the development of rapidly dissolving
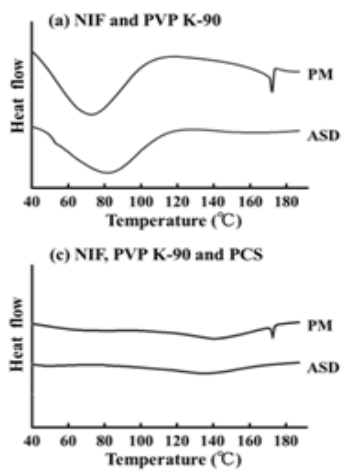
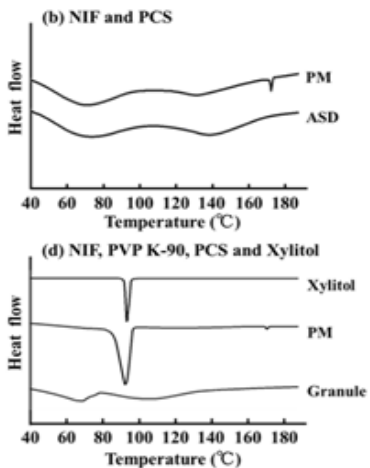

Figure 7: Differential scanning calorimetry (DSC) plots of the physical mixture (PM), adsorption solid dispersion (ASD), and granules. (a) Nifedipine (NIF) and polyvinylpyrrolidone (PVP) K-90 (1:4); (b) NIF and porous calcium silicate (PCS) (1:8); (c) NIF, PVP K-90 and PCS (1:4:8); and (d) NIF, PVP K-90, PCS, and xylitol (1:4:8:14).

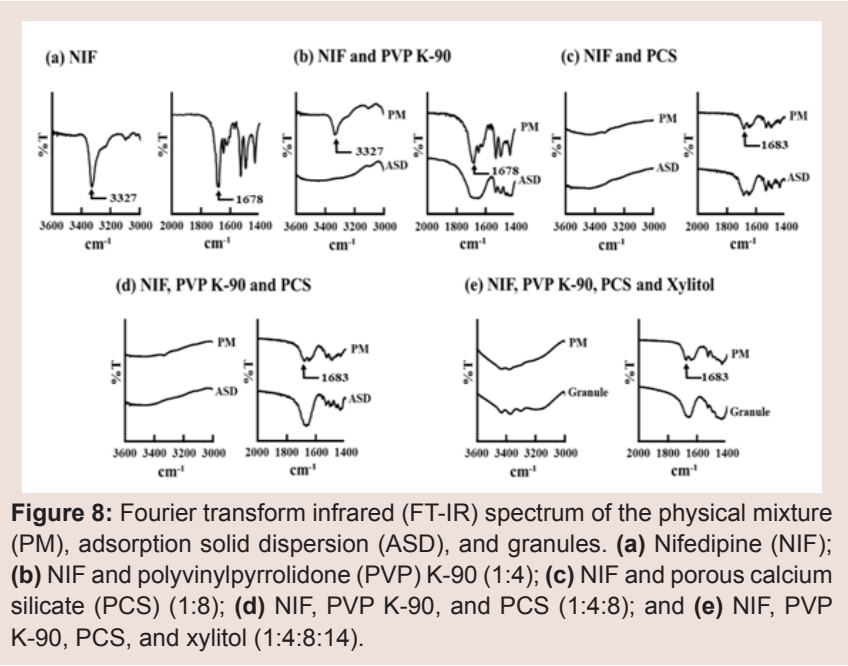


Citation: Uegaki Y, Hirai N, Takatani-Nakase T, Takahashi K. Development of Controlled-Release Solid Dispersion Granules Containing a Poorly WaterSoluble Drug, Porous Calcium Silicate, and the Water-Soluble Polymer Polyvinylpyrrolidone. J Pharmaceu Pharmacol. 2018; 6(1): 7.

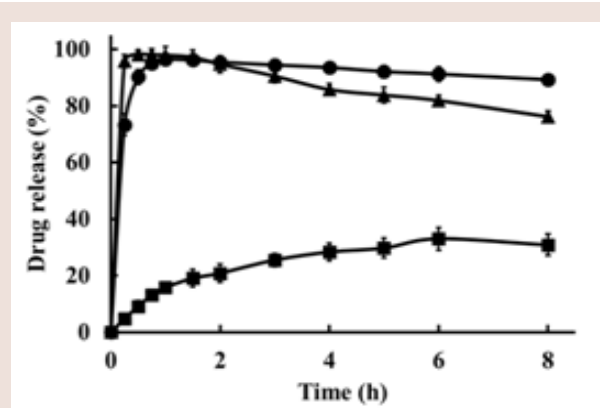

Figure 9: Effect of the addition of polyvinylpyrrolidone (PVP) on the dissolution

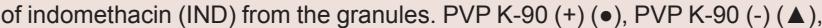
IND ( $\bullet)$. Data are presented as the mean \pm SD $(n=3-5)$.

granules of IND containing PCS [26]. To further investigate the sustained release effect of the combination of PVP K-90 and PCS, granules containing IND were prepared. As shown in Figure 9, sustained release of the drug was not observed.

To investigate the differences between IND and NIF, the physical characteristics of the IND-containing granules were investigated. The representative PXRD peaks of IND were observed at $11.7^{\circ}, 16.7^{\circ}$ and $21.8^{\circ}$ (Figure 10). These PXRD peaks and endothermic peaks of DSC plots derived from the drug crystals of IND disappeared in the ASD (Figure 11). These findings suggested that IND exists in an amorphous state in the PCS formulation [7,15,17,21-23,43]. PXRD peaks derived from the crystals of IND disappeared in the PCS formulation similarly as observed in the ASD, but the derived from xylitol crystals did not completely disappear. In the same manner, the endothermic peaks of DSC plots derived from IND crystals also disappeared in the PCS formulation. These findings suggest that IND exists in amorphous states in PCS formulations.

FT-IR spectra were analyzed to determine the interactions in IND formulations (Figure 12). The IR spectrum of pure IND displayed characteristic peaks at 1717 (acid $\mathrm{C}=\mathrm{O}$ stretching) and $1692 \mathrm{~cm}^{-1}$ (benzoyl $\mathrm{C}=\mathrm{O}$ stretching) as shown in Figure 12a. These peaks were observed in the spectra of PMS containing IND and PVP K-90 or PCS (Figures 12b and 12c). Conversely, ASD samples containing IND and PVP K-90 or PVP displayed a new absorption peak at $1684 \mathrm{~cm}^{-1}$ (Figures 12b and 12c). Fujii M et al. and Taylor LS et al. reported that IND solid dispersions containing PVP or crospovidone exhibit absorption at $1684 \mathrm{~cm}^{-1}[43,44]$. These results suggest that a hydrogen bond between the acid carboxyl group of IND interacts with the amide carbonyl group of PVP. However, as PCS does not contain an amide carbonyl group in its structure, the new absorption peak at $1674 \mathrm{~cm}^{-1}$ in this study does not correspond to the interaction (Figure $12 b)$. Bahl D et al. reported an ion-dipole interaction (rather than salt formation) between $\mathrm{Mg}^{2+}$ and/or $\mathrm{Al}^{3+}$ ions at the surface of magnesium aluminometasilicate (Neusilin US2) and the carboxyl group of IND [15]. They also suggested the presence of a hydrogen bond between the carboxyl group of IND and the silanol group of Neusilin US2 [15]. Doan V et al. reported that the carbonyl band of - $\mathrm{COOH}$ at 1775 $\mathrm{cm}^{-1}$ shifts to $1676 \mathrm{~cm}^{-1}$ upon conversion to the $\mathrm{Na}$ salt form [45]. From these results, it is considered that IND interacts with PCS via salt formation between the COO- moiety of IND and $\mathrm{Ca}^{2+}$ of PCS. Furthermore, the disappearance of the peak for the benzoyl carbonyl (a)

(b)
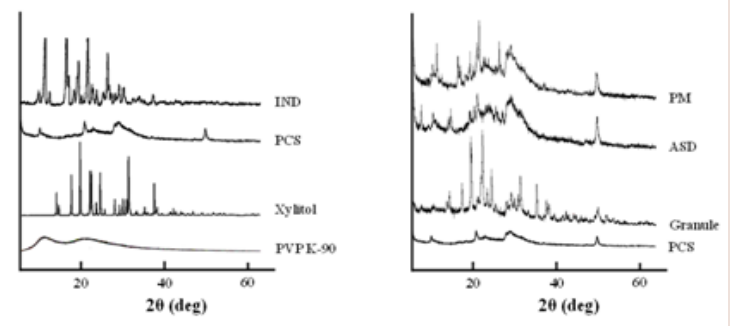

Figure 10: Powder X-ray diffraction (PXRD) patterns of (a) Indomethacin (IND), porous calcium silicate (PCS), xylitol, and polyvinylpyrrolidone (PVP) K-90. (b) Physical mixture (PM) (IND, PVP K-90, PCS and xylitol; 1:2:4:7), adsorption solid dispersion (ASD), and granules.

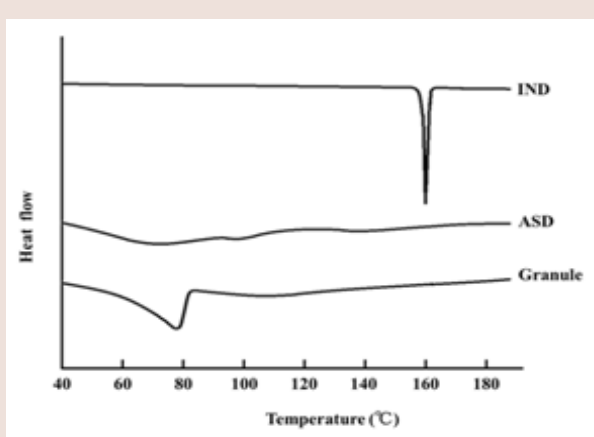

Figure 11: Differential scanning calorimetry (DSC) plots of indomethacin (IND), physical mixture (PM) (IND, PVP K-90, PCS and xylitol; 1:2:4:7), adsorption solid dispersion (ASD), and granules.

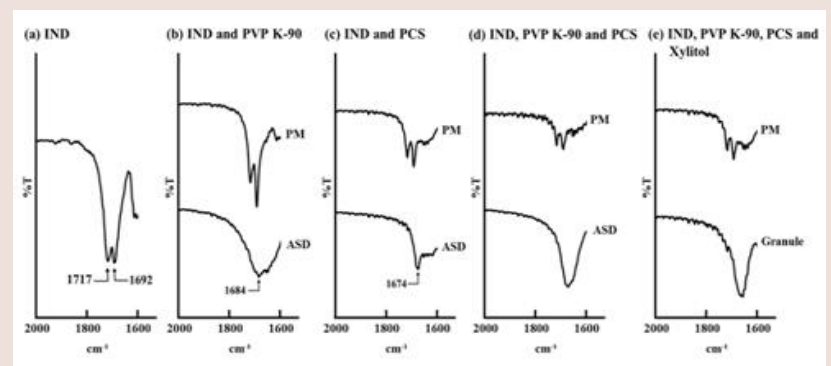

Figure 12: Fourier transform infrared (FT-IR) spectrum of the physical mixture (PM), adsorption solid dispersion (ASD), and granules. (a) Indomethacin (IND); (b) IND and polyvinylpyrrolidone (PVP) K-90 (1:2); (c) IND and porous calcium silicate (PCS) (1:4); (d) IND, PVP K-90, and PCS (1:2:4); and (e) IND, PVP K-90, PCS, and xylitol (1:2:4:7).

group at $1692 \mathrm{~cm}^{-1}$ suggested hydrogen bonding between the benzoyl carbonyl and silanol groups of PCS. Similar results were obtained for the PM and ASD samples containing IND, PVP K-90, and PCS or IND, PVP K-90, PCS, and xylitol (Figures 12d and 12e). These results suggest that IND and PVP interact via hydrogen bonding and that IND and PCS interact via salt formation in the granules. As mentioned previously, NIF interacts with PVP K-90 and PCS via hydrogen bonding. Thus, hydrogen bonding among the drugs, PVP K-90, and PCS may be important for sustained drug release.

Fan $\mathrm{C}$ et al. reported sustained drug release from PVP K-30 surface-coated beads [46]. In their study, the drug did not interact with PVP K-60, and they suggested that the gelling, clumping, 
Citation: Uegaki Y, Hirai N, Takatani-Nakase T, Takahashi K. Development of Controlled-Release Solid Dispersion Granules Containing a Poorly WaterSoluble Drug, Porous Calcium Silicate, and the Water-Soluble Polymer Polyvinylpyrrolidone. J Pharmaceu Pharmacol. 2018; 6(1): 7.

and agglomeration of PVP K-30 surface-coated beads resulted in slow release of the drug. In this study, NIF, but not IND, exhibited sustained release from PVP K-90-containing granules. These results suggest that the sustained release of NIF is due to hydrogen bonding among NIF, PVP K-90, and PCS and not simple surface coating.

\section{Conclusion}

The release rates of NIF from granules decreased as the molecular weight of PVP and the content of PVP K-90 in the granules were increased. However, IND was rapidly released from granules containing PCS and PVP K-90. The DSC and PXDR results indicated a significant loss of crystallinity of NIF and IND in the granules. The FT-IR results for NIF formulations indicated the existence of hydrogen bonds between NIF and PVP and between NIF and PCS. Moreover, the existence of a hydrogen bond between PVP K-90 and PCS was confirmed. Although a hydrogen bond likely exists between IND and PVP, IND likely interact with PCS via salt formation. The results of this study suggest that hydrogen bonding among a poorly water-soluble drug, PCS, and PVP K-90 mainly contributed to this controlled system.

\section{References}

1. Amidon GL, Lennernas H, Shah VP, Crison JR (1995) A theoretical $\square$ basis for a biopharmaceutic drug classification: the correlation of in vitro drug product dissolution and in vivo bioavailability. Pharm Res 12: 413-420.

2. Desai D, Kothari S, Chen W, Wang J, Huang M, et al. (2011) Fatty acid and water-soluble polymer-based controlled release drug delivery system. J Pharm Sci 100: 1900-1912.

3. Lipinski C (2002) Poor aqueous solubility-an industry wide problem in drug discovery. Am Pharm Rev 5: 82-85.

4. Timpe C (2007) Strategies for formulation development of poorly watersoluble drug candidates-a recent perspective. Am Pharm Rev 10: 104-109.

5. Hwang I, Kang CY, Park JB (2017) Advances in hot-melt extrusion technology toward pharmaceutical objectives. J Pharm Invest 47: 123-132.

6. Shah S, Maddineni S, Lu J, Repka MA (2013) Melt extrusion with poorly soluble drugs. Int J Pharm 453: 233-252.

7. Moneghini M, Kikic I, Voinovich D, Perissutti B, Filipović-Grcić J (2001) Processing of carbamazepine-PEG 4000 solid dispersions with supercritical carbon dioxide: preparation, characterisation, and in vitro dissolution. Int $J$ Pharm 222: 129-138

8. Betageri GV, Makarla KR (1995) Enhancement of dissolution of glyburide by solid dispersion and lyophilization techniques. Int J Pharm 126: 155-160.

9. Maniruzzaman M, Rana MM, Boateng JS, Mitchell JC, Douroumis D (2013) Dissolution enhancement of poorly water-soluble APIs processed by hot-melt extrusion using hydrophilic polymers. Drug Dev Ind Pharm 39: 218-227.

10. Pasquali I, Bettini R, Giordano F (2008) Supercritical fluid technologies: an innovative approach for manipulating the solid-state of pharmaceuticals. Adv Drug Deliv Rev 60: 399-410.

11. Hu J, Johnston KP, Williams RO $3^{\text {rd }}$ (2004) Nanoparticle engineering processes for enhancing the dissolution rates of poorly water soluble drugs. Drug Dev Ind Pharm 30: 233-245.

12. Leuner C, Dressman J (2000) Improving drug solubility for oral delivery using solid dispersions. Eur J Pharm Biopharm 50: 47-60.

13. Takeuchi H, Nagira S, Yamamoto H, Kawashima Y (2005) Solid dispersion particles of amorphous indomethacin with fine porous silica particles by using spray-drying method. Int J Pharm 293: 155-164.

14. Yamashita K, Nakate T, Okimoto K, Ohike A, Tokunaga Y, et al. (2003) Establishment of new preparation method for solid dispersion formulation of tacrolimus. Int J Pharm 267: 79-91.
15. Bahl D, Bogner RH (2006) Amorphization of indomethacin by co-grinding with neusilin US2: amorphization kinetics, physical stability and mechanism. Pharm Res 23: 2317-2325

16. Knopp MM, Olesen NE, Holm P, Langguth P, Holm R, et al. (2015) Influence of polymer molecular weight on drug-polymer solubility: a comparison between experimentally determined solubility in PVP and prediction derived from solubility in monomer. J Pharm Sci 104: 2905-2912.

17. Lehmkemper K, Kyeremateng SO, Bartels M, Degenhardt M, Sadowski G (2018) Physical stability of API/polymer-blend amorphous solid dispersions. Eur J Pharm Biopharm 124: 147-157.

18. Bhardwaj V, Trasi NS, Zemlyanov DY, Taylor LS (2018) Surface area normalized dissolution to study differences in itraconazole-copovidone solid dispersions prepared by spray-drying and hot melt extrusion. Int $\mathrm{J}$ Pharm 540: 106-119.

19. Hanada M, Jermain SV, Williams RO $3^{\text {rd }}$ (2018) Enhanced dissolution of a porous carrier-containing ternary amorphous solid dispersion system prepared by a hot melt method. J Pharm Sci 107: 362-371.

20. Xia Y, Yuan M, Deng Y, Ke X, Ci T (2017) Different effects of silica added internal or external on in vitro dissolution of indomethacin hot-melt extrudates. Int J Pharm 534: 272-278

21. Ozeki T, Takashima Y, Nakano T, Yuasa H, Kataoka M, et al. (2011) Preparation of spray-dried microparticles using Gelucire 44/14 and porous calcium silicate or spherical microcrystalline cellulose to enhance transport of water-insoluble pranlukast hemihydrate across Caco-2 monolayers. Adv Powder Technol 22: 623-628.

22. Sharma S, Sher P, Badve S, Pawar AP (2005) Adsorption of meloxicam on porous calcium silicate: characterization and tablet formulation. AAPS PharmSciTech 6: E618-E625.

23. Kinoshita M, Baba K, Nagayasu A, Yamabe K, Shimooka T, et al. (2002) Improvement of solubility and oral bioavailability of a poorly water-soluble drug, TAS-301, by its melt-adsorption on a porous calcium silicate. J Pharm Sci $91: 362-370$.

24. Yuasa H, Takashima Y, Kanaya Y (1996) Studies on the development of intragastric floating and sustained release preparation. I. Application of calucium silicate as a floating carrier. Chem Pharm Bull 44: 1361-1366.

25. Fijimoto Y, Hirai N, Takatani-Nakase T, Takahashi K (2016) Preparation and evaluation of solid dispersion tablets by a simple and manufacturable wet granulation method using porous calcium silicate. Chem Pharm Bull (Tokyo) 64: 311-318.

26. Fujimoto Y, Hirai N, Takatani-Nakase T, Takahashi K (2016) Novel tablet formulation of amorphous indomethacin using wet granulation with a highspeed mixer granulator combined with porous calcium silicate. J Drug Deliv Sci Technol 33: 51-57.

27. Tran HT, Park JB, Hong KH, Choi HG, Han HK, et al. (2011) Preparation and characterization of $\mathrm{pH}$-independent sustained release tablet containing solid dispersion granules of a poorly water-soluble drug. Int J Pharm 415: 83-88.

28. Dereymaker A, Scurr DJ, Steer ED, Roberts CJ, Van den Mooter G (2017) Controlling the release of indomethacin from glass solutions layered with a rate controlling membrane using fluid-bed processing. Part 1: Surface and cross-sectional chemical analysis. Mol Pharm 14: 959-973.

29. Hasan El, Amro BI, Arafat T, Badwan AA (2003) Assessment of controlled release hydrophilic matrix formulation for metoclopramide $\mathrm{HCl}$. Eur J Pharm Biopharm 55: 339-344.

30. Abdel-Rahman SI, Mahrous GM, El-Badry M (2009) Preparation and comparative evaluation of sustained release metoclopramide hydrochloride matrix tablets. Saudi Pharm J 17: 283-288.

31. Kumar S, Pandey M, Saraf SA (2009) Novel sustained release gastroretentive floating matrix tablets of acyclovir: formulation and in vitro evaluation. J Pharm Res 2: 717-722.

32. Tran PH, Tran TT, Park JB, Lee BJ (2011) Controlled release systems containing solid dispersions: strategies and mechanisms. Pharm Res 28: 2353-2378. 
Citation: Uegaki Y, Hirai N, Takatani-Nakase T, Takahashi K. Development of Controlled-Release Solid Dispersion Granules Containing a Poorly WaterSoluble Drug, Porous Calcium Silicate, and the Water-Soluble Polymer Polyvinylpyrrolidone. J Pharmaceu Pharmacol. $2018 ; 6(1): 7$.

33. Sun Y, Tao J, Zhang GG, Yu L (2010) Solubilities of crystalline drugs in polymer: An improved analytical method and comparison of solubilities of indomethacin and nifedipine in PVP, PVP/VA, and PVAc. J Pharm Sci 99: 4023-4031.

34. Korsmeyer RW, Lustig SR, Peppas NA (1986) Solute and penetrant diffusion in swellable polymers. I. Mathematical modeling. J Polym Sci Part B: Polym Phys 24: 395-408.

35. Ritger PL, Peppas NA (1987) A simple equation for description of solute release I. Fickian and non-fickian release from non-swellable devices in the form of slabs, spheres, cylinders or discs. J Control Release 5: 23-36.

36. Ritger PL, Peppas NA (1987) A simple equation for description of solute release II. Fickian and anomalous release from swellable devices. J Control Release 5: 37-42.

37. Salome AC, Godswill CO, Ikechukwu IO (2013) Kinetics and mechanisms of drug release from swellable and non swellable matrices: a review. Res J Pharm Biol Chem Sci 4: 97-103.

38. Buzzi V, Brudner M, Wagner TM, Bazzo GC, Pezzin AP, et al. (2013) Caboxymetylcellulose/Gelatin blends loaded with piroxicam: preparation, characterization and evaluation of in vitro release profile. J Encapsul Adsorp Sci 3: 99-107.

39. Abdelbary GA, Tadros MI (2008) Design and in vitro/in vivo evaluation of novel nicorandil extended release matrix tablets based on hydrophilic interpolymer complexes and a hydrophobic waxy polymer. Eur J Pharm
Biopharm 69: 1019-1028.

40. Siepmann J, Peppas NA (2001) Modeling of drug release from delivery systems based on hydroxypropyl methylcellulose (HPMC). Adv Drug Deliv Rev 48: 139-157.

41. Forster A, Hempenstall J, Rades T (2001) Characterization of glass solutions of poorly water-soluble drugs produced by melt extrusion with hydrophilic amorphous polymers. J Pharm Pharmacol 53: 303-315.

42. Cilurzo F, Selmin F, Minghetti P, Gennari CG, Demartin F, et al. (2008) Characterization and physical stability of fast-dissolving microparticles containing nifedipine. Eur J Pharm Biopharm 68: 579-588.

43. Fujii M, Okada H, Shibata Y, Teramachi H, Kondoh M, et al. (2005) Preparation, characterization, and tableting of a solid dispersion of indomethacin with crospovidone. Int J Pharm 293: 145-153.

44. Taylor LS, Zografi G (1997) Spectroscopic characterization of interactions between PVP and indomethacin in amorphous molecular dispersions. Pharm Res 14: 1691-1698.

45. Doan V, Koppe R, Kasai PH (1997) Dimerization of carboxylic acids and salts: an IR study in perfluoropolyether media. J Am Chem Soc 119: 9810-9815.

46. Fan C, Pai-Thakur R, Phuapradit W, Zhang L, Tian H, et al. (2009) Impact of polymers on dissolution performance of an amorphous gelleable drug from surface-coated beads. Eur J Pharm Sci 37: 1-10. 